\title{
Erratum
}

\section{A Novel Approach to Monobenzannulated Spiroketals Using Styrenes in the Kulinkovich Reaction}

Isabell Haym, Margaret A. Brimble* Synlett 2009, 2315.

In the advanced online (e-First) publication of this manuscript, the structure of compound 33 was incorrect. It has been corrected for both the print and the current online versions. We apologize for this mistake. 